\title{
Correlation between psychophysiological response and hospital management model for nurses involved in medication errors
}

\author{
Lin-Lin Li ${ }^{1,2}$, Yu-Chun Yin ${ }^{2}$, Po-Erh Liu ${ }^{3}$, Chen-Shu Ling ${ }^{3}$, Cheng-Yu Kuo ${ }^{4}$, Hsien-Wen Kuo *5,6 \\ ${ }^{1}$ Department of Nursing, Tali Jen-Ai Hospital, Taichung, Taiwan \\ ${ }^{2}$ School of Nursing, Chung Shan Medical University, Taichung, Taiwan \\ ${ }^{3}$ School of Nursing, HungKuang University, Taichung, Taiwan \\ ${ }^{4}$ College of Arts and Sciences, University of San Francisco, United States \\ ${ }^{5}$ Institute of Environmental and Occupational Health Sciences, National Yang-Ming University, Taipei, Taiwan \\ ${ }^{6}$ Public Health, National Defense Medical Center, Taipei, Taiwan
}

Received: February 16, 2017

DOI: $10.5430 /$ jnep.v7n8p72
Accepted: March 9, 2017

Online Published: March 16, 2017

\begin{abstract}
Because hospitals in Taiwan now place a lot of emphasis on hospital accreditation, leading to a heavy workload for nurses, nurses are unintentional involved in patient medication errors (MEs). In this research, we explore the possible correlation between psychophysiological responses of nursing staff and a hospital management model regarding MEs. We conducted a cross-sectional study design in one hospital in central Taiwan. A total of 345 nurses at Tali Jen-Ai Hospital 1 were selected. A questionnaire was chosen as the study instrument. The questionnaire asked the following information: basic data, classification of MEs, frequency and severity of psychophysiological responses, and information about the hospital's management model and support system. We found that the hospital management model had a significant negative correlation with the frequency of physiological symptoms $(r=-0.14, p<.01)$. Likewise, the support system also had a substantial negative correlation with the scores of the physiological symptom, behavioral responses, and psychophysiological responses. Using multiple regression analysis adjusted for work duration, job title, and degree of injury, we found that the support system was significantly correlated with the nurses' psychophysiological responses. Both the hospital management and the support system decreased the psychophysiological responses of the nurses. Therefore, we recommend that supervisors implement a management strategy to deal specifically with MEs. When nurse managers have frequently empathy and no-blame attitude to replace disrespectful or skeptical ways to nurse with ME, they will empower their enthusiasm and responsibility to improve the reduction of ME.
\end{abstract}

Key Words: Incidents, Psychophysiological response, Hospital management model, Support system

\section{INTRODUCTION}

Administering medication is an important nursing task. A busy work schedule, combined with increased numbers and dosages of prescribed medications, puts nurses at risk of mak- ing serious medication errors (MEs). ${ }^{[1]}$ A reduction of MEs and an improvement of patient safety are two of the most important issues in international health care today. The report "Quality and Safe Use of Medicines", allows us to raise the

\footnotetext{
*Correspondence: Hsien-Wen Kuo; Email: hwkuo@ym.edu.tw; Address: No.155, Sec.2, Li-Nong Street, Taipei, 112 Taiwan (ROC), Institute of Environmental and Occupational Health Sciences, National Yang-Ming University, Taipei, Taiwan.
} 
opportunity to address some of the safety-related issues with a view toward enhancing overall patient safety. ${ }^{[2]}$ Although MEs are an inevitable consequence of human performance, America spend greatly expenditures on preventable MEs annually, which requires building on successful practices and policy. In 2007, preventable inpatient and outpatient MEs costs in US were estimated to be approximately $\$ 16.4$ billion and $\$ 4.2$ billion, respectively. It is also estimated that there are 3.8 million inpatient admissions and 3.3 million outpatient hospital visits each year in the U.S. ${ }^{[3]}$ Because of the considerable number of deaths and financial loss caused by MEs, countries around the world are exceptionally aware of the importance of patient safety. Consequently, government organizations are pouring more resources into research in this area to improve the safety of patients. In an ME event in Taiwan in 2002, injecting patients with the wrong medication resulted in the death of one and the injury of seven others. In another ME event in Taiwan, patients were injected with a hypoglycemic agent instead of medication to treat cold symptoms, resulting in the death of one infant and the injury of another 114 infants. As a result reports such as these, patient safety began to attract wider public attention. ${ }^{[4]}$ Taiwan's government established a committee regarding patient safety in February 2003, promoting safety-related plans and activities that included putting the "Guidelines for Clinical Safety Procedures" into place, which guides medical institutions to establish patient safety committees, implement education training for staff, establish "patient-centered safety health care" criteria, create an incident reporting systems, ${ }^{[5]}$ and provides a listing of patient safety items as part of a newly introduced hospital accreditation system. Consequently, these measures have caused hospitals to focus more intently on the issue of patient safety and to implement these and other important programs accordingly.

When nurses make MEs, they face tremendous pressure. Hence, long-standing exposure to stressful events such as MEs can lead to negative feelings, including long-term anxiety, sense of helplessness, social withdrawal, and apathetic attitudes. These psychological results, in turn, have adverse effects on the nurses' self-confidence and self-esteem. ${ }^{[6]} \mathrm{Be}$ cause serious MEs can have a great impact on nurses, both personally and professionally, nurses involved in MEs are often fired or rashly blame by their supervisors. ${ }^{[7,8]}$ Health care organizations usually find a scapegoat when an ME occurs. Such punitive attitudes not only leave the underlying problems unresolved but also conceal more errors within the organizational system, thereby exposing patients to more health care risks. ${ }^{[9]}$ Hospitals should have less punitive attitudes and no-blame culture toward MEs in order to reduce MEs from happening in the future. ${ }^{[10]}$ Limited studies were

Published by Sciedu Press assessed that psychophysiological responses in nurses with ME correlated with hospital management and supporting system. Possibly, if nurses with ME are empathized with supporting system from colleagues who work in those hospital unit rather than disrespectful and blameful, zero ME reduction may be successfully achieved by no-blame culture and climate of supporting system. If hospitals have a well-organized supporting system for their nurses, patients are more likely to avoid further injury. Nurses involved in MEs can learn more from their mistakes and avoid making the same mistake in the future. It is easily approach to the win-win policy in medical services. Therefore, hospital supervisors should empathy the medical errors in nurses with $\mathrm{ME}$ and get together to elaborate the reasons of ME, but instead, provide more supporting system to alleviate their psychophysiological responses.

\subsection{The aim of this study}

The purpose of this study is to investigate the frequency and severity of psychophysiological response symptoms among nurses and to assess the correlation between a hospital management model and its support system.

\subsection{Hypothesis}

There are a heightened frequency and severity of psychophysiological responses associated with the hospital management model and the support system among nurses with MEs.

\section{SubJECTS AND METHODS}

\subsection{Study design and participant selection}

We conducted a cross-sectional study in Tali Jen-Ai hospital in central Taiwan. We surveyed 360 nurses regarding their past experiences of MEs and their views on the issue. The response rate was $99.4 \%$.

\subsection{Design of questionnaire}

Medication error is frequently unpreventable or controllable during the complicated medical prescription now. According to Taiwan's Joint Commission of Taiwan (JCT) defined medical incident included medical error, medical adverse events and sentinel events. ${ }^{[11]}$ This study defined ME is 'a failure in the treatment process that leads to, or has the potential to lead to, harm to the patient'. Based on hospital regulation in Taiwan, nurses with ME should immediately and actively report to nurse manger. Nurse with ME also were punished or seriously warned according to severity of the potential to harm to the patient, such as prescribing faults, prescription errors, falls with patients, and mistakes that result from applying bad rules, or misapplying or failing to apply good rules etc. The questionnaire was based on relevant literature and the findings of 10 in-depth interviews of nurses. The 
psychophysiological responses and the hospital management model's scale were drafted, and the subsequently reviewed and revised by five medical specialists. The physical and psychological response scale was divided into three domains, which totally consisted of 20 questions. The first domain covered psychological symptoms. These included depression, uneasiness, helplessness, a sense of guilt, grief, and a loss of self-confidence. The second domain focused on physiological symptoms.

These consisted of dizziness, gastrointestinal discomfort, and lower back pain. The third domain evaluated behavioral responses. These included lack of appetite, sleep disorders, fatigue, and attention deficit issues. A Likert scale was adopted as the scoring system. The symptom frequency answers were: "always", "sometimes" or "seldom". These answers were given scores of 3, 2, and 1 point(s), respectively. The higher the score, the more frequent the responses were. Regarding the severity of the symptoms, the possible answers were: "extremely severe", "severe", "moderate" and "mild". These answers were also given scores of 4, 3, 2, and 1 point(s), respectively. Consequently, the higher the score, the more serious the responses were. The content validity index (CVI) was between .90-.92. The internal consistency reliability for Cronbach's $\alpha$ coefficient was 0.77 . The evaluation of the hospital management model and the support system consisted of 14 questions. These included proactive assistance, workload, education and training, counseling hotline, and psychological counseling. It was scored on a five-point Likert scale. The possible answers were: "strongly agree", "agree", "neither agree nor disagree", "disagree" and "strongly disagree". The answers were given $5,4,3,2$, and 1 point(s), respectively.
Thus, nurses who had high scores received more support from their hospital supervisors. The average CVI was .90 while the internal consistency reliability for Cronbach's $\alpha$ coefficient was .83 .

\subsection{Statistical analysis}

The questionnaires went through file coding before statistical analysis with the SPSS 10.0/PC software package (SPSS Inc., Chicago, IL, 2011). Descriptive statistics were used to describe the demographic information and the characteristics of nurses with MEs, the frequency and severity of psychophysiological responses, the hospital management model, and the support system. Pearson's correlation was used to assess the connection between the hospital management model, the support system, and the psychophysiological responses of the nurses. Multiple regression analysis was used to evaluate the major predictive factors of the psychophysiological responses among the nurses.

\section{RESUlts}

Half of the participants were between 26 to 30 years old, and $66.4 \%$ had graduated from at least junior college. Two-thirds of the nurses had no religious beliefs, and over $80 \%$ were single. $32.6 \%$ of the nurses had one to three years' experience working at that particular hospital. The departments that had the most MEs were internal medicine, followed by the Intensive Care Unit (ICU): $44.9 \%$ of the MEs occurred in the internal medicine department, and $29.9 \%$ of the MEs took place in the ICU. The lowest occurrences of MEs were in pediatrics $(2.4 \%)$ and the outpatient clinic $(0.9 \%)$. As shown in Table 1, nurse practitioners had the highest occurrence of MEs $(77.7 \%)$.

Table 1. Demographic information of the nurses $(\mathrm{N}=345)$

\begin{tabular}{|c|c|c|c|c|c|}
\hline Variables & $\mathbf{n}$ & $(\%)$ & Variables & $\mathbf{n}$ & $(\%)$ \\
\hline Age (years) & & & Incident Occurrence Unit & & \\
\hline$<25$ & 142 & 41.2 & Outpatient & 3 & 0.9 \\
\hline $26-30$ & 172 & 49.9 & Emergency & 11 & 3.2 \\
\hline$>30$ & 31 & 9.0 & ICU & 103 & 29.9 \\
\hline Education Level & & & Internal Medicine & 155 & 44.9 \\
\hline Junior College & 229 & 66.4 & Surgical & 18 & 5.2 \\
\hline University & 116 & 33.6 & Pediatrics & 5 & 2.4 \\
\hline Religious Belief & & & Operational Room & 2 & 0.6 \\
\hline No & 228 & 66.0 & Obstetrics and Gynecology & 2 & 0.6 \\
\hline Yes & 117 & 34.0 & Medical-Surgical & 42 & 12.2 \\
\hline Work duration (years) & & & Psychiatry & 2 & 0.6 \\
\hline$<1$ & 43 & 12.5 & Hemodialysis & 2 & 0.6 \\
\hline $1-3$ & 117 & 33.9 & Job Title & & \\
\hline $3-7$ & 124 & 35.9 & Nurse & 77 & 22.3 \\
\hline$>7$ & 61 & 17.7 & Nurse Practioner & 268 & 77.7 \\
\hline
\end{tabular}


Table 2 presents the different types of MEs. These include patient falls (38.4\%), self-extubation (22.4\%), accidental removal medical tube (21.8\%), administrative errors (7.8\%), and patient identification errors (3.2\%). The degrees of injury were as follows: mild injury (73.7\%), moderate injury
(25.2\%), and severe injury (1.2\%). The MEs occurred mostly during the day shift (37.7\%), followed by the evening shift (31.3\%). The night shift had the lowest occurrence of MEs (1.4\%). Of the respondents, $95.9 \%$ believed that nurses should not be punished for MEs.

Table 2. Distribution of incident errors $(\mathrm{N}=345)$

\begin{tabular}{|c|c|c|c|}
\hline Variables & Classification & $\bar{n}$ & $(\%)$ \\
\hline \multirow{8}{*}{ Kinds of incident errors } & Medication Administration & 27 & 7.8 \\
\hline & Fall & 132 & 38.4 \\
\hline & Self-Extubation & 77 & 22.4 \\
\hline & Unplanned extubation & 76 & 21.8 \\
\hline & Transfusion errors & 2 & 0.6 \\
\hline & Patient identification errors & 11 & 3.2 \\
\hline & Poor nursing techniques, mishandling & 11 & 3.2 \\
\hline & Other (Including Suicide) & 9 & 2.6 \\
\hline \multirow{3}{*}{ Degree of injury } & Mild (Level < 1) & 254 & 73.7 \\
\hline & Moderate (Level 2 to 3) & 87 & 25.2 \\
\hline & Severe (Level > 4) & 4 & 1.2 \\
\hline \multirow{3}{*}{ Time of Occurrence } & Day Shift & 130 & 37.7 \\
\hline & Evening Shift & 108 & 31.3 \\
\hline & Night Shift & 107 & 01.4 \\
\hline \multirow{2}{*}{$\begin{array}{l}\text { Nurses report their involvement in IE } \\
\text { without being punished }\end{array}$} & Agree & 331 & 95.9 \\
\hline & Disagree & 14 & 04.1 \\
\hline
\end{tabular}

Table 3 shows that for those nurses in ME incidents, the most common physiological symptoms were lower back pain (1.66), dizziness (1.26), and gastrointestinal discomfort (1.24). The most severe physiological symptoms were lower back pain (1.73), gastrointestinal discomfort (1.43), dizziness (1.33), depression (1.57), tense feelings (1.46), and irritability (1.31). The psychological symptoms that occurred most often and that were the most severe were senses of depression and helplessness. The behavioral responses took place most frequently and that were the most severe were fatigue (1.61 and 1.46, respectively). This result was followed by sleep disorder (1.58) and attention deficit (1.23). The behavioral responses that had the lowest scores were the avoidance of joining certain activities (0.71) and sleep disorder (0.58). Therefore, fatigue was the most significant effect of the different behavior responses, while sleep disorder occurred frequently but was not as severe.

Table 4 shows the correlation between the frequency and the severity of the nurses' psychophysiological responses and the hospital management model and support system. The hospital management model showed a significant negative correlation with the frequency of physiological responses $(r$
$=-0.14, p<.01)$. This illustrates that hospital management actions may lower the occurrence of physiological symptoms among nurses involved in MEs. The hospital support system was significantly correlated with the frequency $(r=$ $-0.19, p<.01)$, behavioral responses $(r=-0.10, p<.05)$, and total score of psychophysiological responses $(r=-0.12$, $p<.01)$. However, there was not a significant correlation in the psychophysiological responses between the hospital management model and the support system.

Table 5 shows both the frequency and the severity of psychophysiological responses in nurses who were affected by the hospital management and support system. Here, we used multiple regression analysis, adjusted for work duration, marital status, religious belief, and level of injury. After adjusting for the covariates, we found that there was a negative correlation between the physiological symptoms and the support system $(p<.05)$. The support system showed a level of marginal statistically significance in the behavioral responses $(p=.068)$. There was also a significant negative correlation between the severity of the physiological responses and the support system $(p<.05)$. Simply put, the better the support system, the less serious the psychological symptoms were. 
Table 3. The frequency and severity of the nurses' psychophysiological responses $(\mathrm{N}=345)$

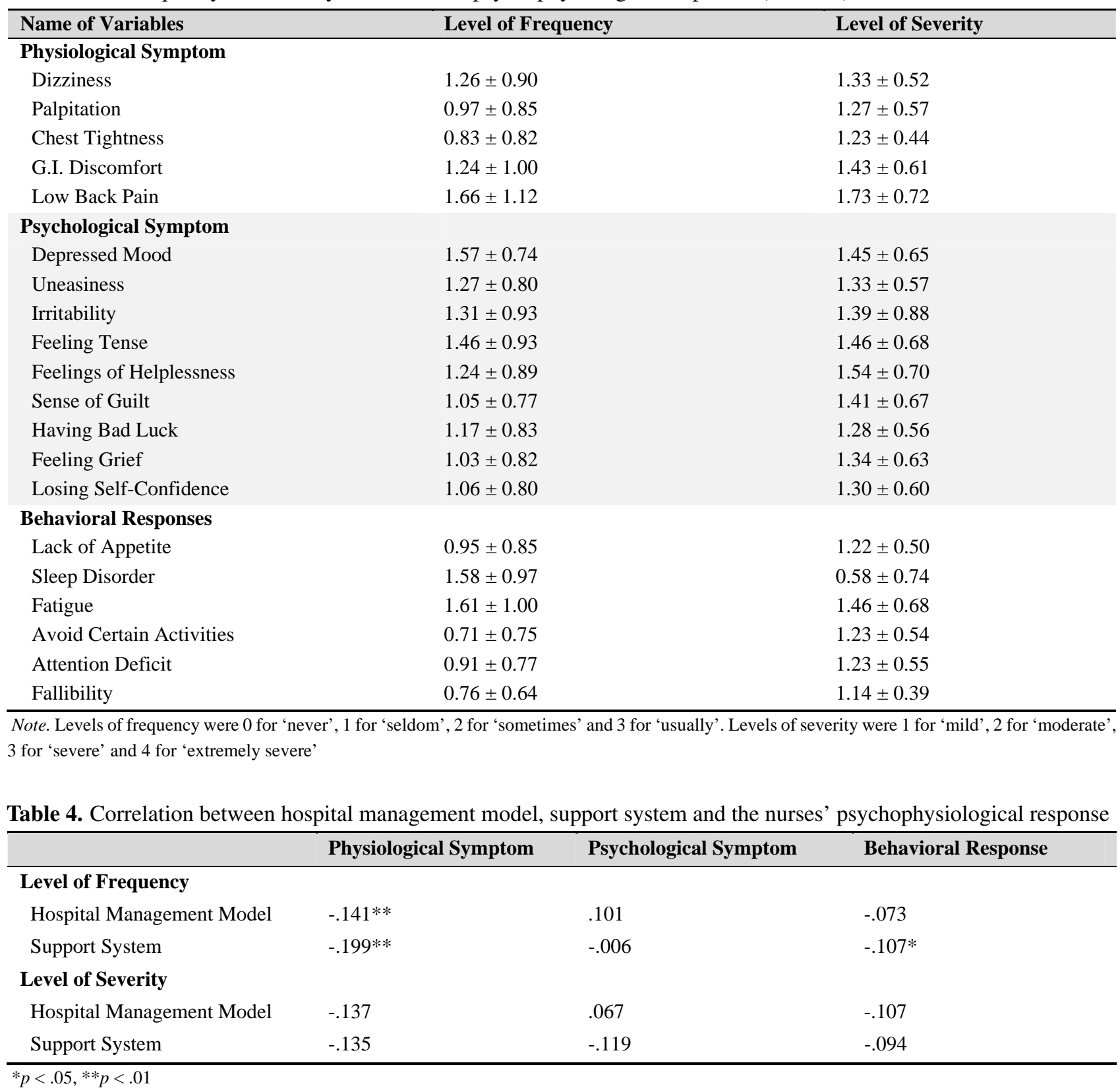

Table 5. Frequency and severity of the nurses' psychophysiological response affected by the hospital management model and support system using multiple regression models

\begin{tabular}{llll}
\hline & Physiological Symptom & Psychological Symptom & Behavioral Response \\
\hline Frequency & & & $-0.073(0.068)$ \\
$\quad$ Hospital Management Model & $-0.150(0.091)$ & $-0.006(0.069)$ & $-0.200(0.109)$ \\
$\quad$ Support System & $-0.424(0.147)^{* *}$ & $-0.056(0.111)$ & $-0.041(0.075)$ \\
Severity & & & $-0.091(0.128)$ \\
$\quad$ Hospital Management Model & $-0.091(0.101)$ & $0.087(0.057)$ & $-0.268(0.099)^{* *}$ \\
$\quad$ Support System & $-0.181(0.179)$ & & \\
\hline
\end{tabular}

Note. Adjustment for work duration, job title, marital status, religious belief and degree of injury. ${ }^{* *} p<.01$ 


\section{Discussion}

One of the accreditation criteria for "patients' rights and safety" in Taiwan has been the handling of MEs. Hospital MEs include patients falling, unexpected extubation, blood transfusion mistakes, improper use of medical equipment, and violent behavior in patients. ${ }^{[11]}$ Although MEs should be actively reported to avoid systemic problems, one study found that the reasons for the inability of health care providers to actively report MEs were a lack of feedback, fear of being penalized when reporting, fear of having one's reputation hurt, and fear of rejection by one's peers. ${ }^{[12]}$ For these reasons, nurses sometimes choose not to report MEs. ${ }^{[13]}$ If the system of reporting MEs is to be successful, the culture and management of hospitals need to be non-punitive, as this significantly contributes as to whether a nurse will responsibly report ME errors. ${ }^{[14]}$ In this study, we found that the most frequent types of MEs were the patient falling, self-extubation, unplanned extubation, and patient identification errors. A prospective cohort study was conducted in 36 U.S. institutions, where they found $19 \%$ of MEs $(605 / 3,216)$ were due to incorrect dosages. In their investigation, the most common types of MEs were giving patients medicine at the wrong times (43\%), forgetting to give patients medicine (30\%), giving patients wrong dosages (17\%), and giving patients unauthorized medicines $(4 \%) .^{[15]}$ In another research, a total of $55(76.4 \%)$ nurses out of a group of 72 believed that more than one factor had contributed to various MEs. Of the contributing factors, the nurses believed that personal negligence $(86.1 \%)$, a heavy workload (37.5\%), and new staff $(37.5 \%)$ were the three main issues. ${ }^{[7]}$ In another study of two departments, the Geriatric Unit (GU) and the Cardiovascular-Thoracic Surgery Unit (CTSU) at Besançon University Hospital (France), they reported that the ME rate was $14.9 \%$. Here, dosage errors were the most frequent types of errors $(41 \%)$, followed by giving patients medicines at the wrong times $(26 \%)$. No potentially life-threatening were observed, however, $8(10 \%)$ were estimated as potentially life-threatening, $20(26 \%)$ potentially significant, and 50 (64\%) potentially minor. ${ }^{[16]}$

MEs in critical care are frequent, serious, and unpredictable. Empirical learning frequently arises from near misses and ME in nursing work, facilitating various preventable strategies to be implemented in place. Culture of safety within hospitals is needed to upgrade the governance in hospital alleviate ME incidence and implement high-quality medical services. ${ }^{[17,18]}$ MEs not only result in patient injuries but also cause extended physiological and psychological effects on nurses. ${ }^{[19]}$ The different behavioral responses of nurses include loss of appetite, fatigue, sleep disorder, and attention deficit disorder. Work stress related to mental fatigue

Published by Sciedu Press is a major risk factor in terms of occupational injuries. The behavioral responses of medical staff to MEs include depression, self-blame, a sense of guilt, grief, helplessness, worry about subsequent problems, and concern over not letting others know. ${ }^{[20]}$ For nurses involved in MEs, lower back pain and gastrointestinal discomfort are the most common psychophysiological symptoms in terms of both frequency and severity. Physiological problems in the nurses involved in MEs often lead to serious feelings of helplessness, blaming oneself, depression, and a sense of guilt. Our study also showed that depression and a sense of helplessness were the most common and severe psychological problems among study population (see Table 3). The results of our work are consistent with previous studies, which also suggests that long-term exposure to stressful events can cause depression, anxiety, feelings of helplessness and satisfaction of life among Iranian employed nurses. ${ }^{[6,20]}$ The behavioral responses that occurred most often and that were the most severe were fatigue, sleep disorder, a lack of appetite, attention deficit problems, and the avoidance of joining certain activities. Individuals under heavy pressure for long periods suffer from insomnia, a lack of appetite, fatigue, and attention deficit issues. ${ }^{[1]}$ Our study also showed that the most severe psychological problem was fatigue, which was caused by work-related pressure and the occurrence of MEs. Nurses' busy schedules often cause fatigue, and this sometimes leads to MEs. In turn, MEs put tremendous pressure on nursing staff, resulting in both emotional problems and physical and psychological fatigue. Therefore, this negative cycle sometimes keeps nurses from telling others of their MEs out of a fear of being punished. ${ }^{[21]}$ Voluntary ME reporting systems can, to some extent, provide meaningful and actionable information to guide patient safety improvement, but these systems' usefulness is limited because of incomplete reporting, underreporting, as well as various other reporting biases. $^{[14,22]}$

Although most nurses must meet various sources of stress in their jobs and with their heavy workloads, MEs, and patient deaths, stress- and/or crisis management-related courses can help nurses maintain positive attitudes. ${ }^{[23]}$ With support activity interventions, such as sharing one's feelings and counseling support groups, nurses are better able to cope with their work-related stresses. ${ }^{[24,25]}$ Therefore, when dealing with MEs, supervisors should not blame nurses. Instead, supervisors should show greater attention to the underlying problems as well as provide counseling support groups to improve the physical and mental well-being of their nurses. The relationship between the hospital management model and nurses' psychophysiological responses shows a negative correlation, in addition to a statistical difference. Our work 
shows that nurses need to be provided with courses and continuing education related to pressure-crisis management for their emotional health. The total scores of the support system and the psychophysiological response symptoms-with the exceptions of the psychological responses-were negatively correlated and had a statistical difference.

As it stands today, too many nurses involved in MEs feel embarrassed and are likely to keep quiet about the incidents out of fear of losing their jobs. ${ }^{[26]}$ Meanwhile, it has been shown that providing counseling for nurses involved in MEs greatly reduces pressure. Effective medication governance included the reporting of ME and counselling system help reduce nurses' emotional stress and preventing ME again to keep patients more safely. ${ }^{[25]}$ Thus, nurse manager should not seriously blame nurses involved in MEs, nor should they adopt punitive ways toward MEs, even in cases of fatal events. ${ }^{[27]}$ Contrary, if nurse manager frequently use empathy and no-blame to replace by punishment to skeptical attitude to nurse with ME, these experiences may empower their professional and responsibility to alleviate ME events again. Moreover, nurses with ME were empathized with the colleagues who work in those hospital unit rather than disrespectful and blameful. We should excuse their active errors because they are human. Eventually, we take for grant that our forgiving and empathy for nurses with ME create the no-blame culture and climate of supporting system, minimal ME incident is successfully achieved for nurse with ME who should not allow to occur mistake in work again.

In the current study, the hospital management model had a significant negative correlation with the frequency of physiological symptoms $(r=-0.14, p<.01)$. The hospital support system had a significant negative correlation with the total scores of physiological symptom frequency, behavioral responses, and psychophysiological responses. Both the management model and the precautionary system that used psychometric testing decreased the psychophysiological responses in the nurses. It is, therefore, obvious that hospital supervisors should assist nurses with a clear and transparent plan for dealing with nursing MEs, which will aid all concerned. ${ }^{[28]}$ Also, using various high-tech equipment, such as mobile device app or computerized physician order entry (CPOE) system, have developed to reduce ME due to inaccessible time to drug delivery or workload-caused fatigue in medical staffs. ${ }^{[29,30]}$
There were some limitations in this cross-sectional study design. First, some nurses involved in MEs at the hospital under investigation had either already been fired or had quit their jobs. Therefore, the nurses who participated in our study may not have adequately represented all nurses involved in MEs. Consequently, our study may have underestimated the frequency of MEs. However, this did not affect the relationship between the hospital management model and the nurses' psychophysiological responses. Second, the nurses' psychophysiological responses were subjective, and thus, influenced by their own personalities and viewpoints. Except for reevaluation of ensure competence among nurses with ME, the effective strategies are wildly initiated to elaborated the mechanism and rooted cause of ME through openness and accurate reporting of ME. Surely, we believe it is important to establish an intervention program that not only provide counseling for nurses to reduce the psychophysiological responses, also is the root cause of ME straightforwardly discovered by the assistance of nurse with ME. ${ }^{[31]}$

\section{CONCLUSIONS AND CLINICAL IMPLICA- TIONS}

We conclude that the organization management model for MEs and the provision of support systems have significant effects on nurses' psychophysiological responses. Therefore, creating a sound ME management model and providing nurses with timely support and positive feedback can be beneficial to nurses. In clinical practice, it is suggested that hospitals provide both crisis management and continuing educational programs for nurses to deal with MEs. Although MEs are unpleasant events full of heightened pressure, positive steps that are put in place by both hospital management and supporting systems can decrease the psychophysiological responses in nurses. Therefore, we suggest that administrators and supervisors put strong management strategies into place to deal efficiently and productively with hospital MEs.

\section{ACKNOWLEDGEMENTS}

This research was supported by the fund of Department of Nursing, Jen-Ai Hospital and all hospital managers were for kindly help on survey work. Special thanks to nurses who participated in the study.

\section{CONFlicts OF INTEREST Disclosure}

The authors declare that there is no conflict of interest.

[2] McBride-Henry K, Foureur M. Medication administration errors: understanding the issues. Aust J Adv Nurs. 2006; 23: 33-41. PMid: 16568877

[3] NEHI. How Many More Studies Will It Take? A Collection of Evi- 
dence That Our Health Care System Can Do Better. Available from: http://www.nehi.net/publications/30/

[4] Chiou ST. Views of patients and the public regarding physician disclosure of medical errors-a review of empirical literature. Taiwan J Public Health. 2007; 26: 339.

[5] Hou SM, Chen HH. New efforts in promoting patient safety. Formos J Med. 2004; 8: 504-9.

[6] Wan YL. Work stress and coping. Nankai J. 2002; 7: 13-9.

[7] Tang FI, Sheu SJ, Yu S, et al. Nurses relate the contributing factors involved in medication errors. J Clin Nur. 2007; 16: 447-57. PMid:17335520 https://doi.org/10.1111/j.1365-2702.20 $05.01540 . x$

[8] Chen YC. Building a patient-centered safe healthcare environment. J Veterans General Hospital. 2003; 70: 2-9.

[9] Berwick DM. Continuous improvement as an ideal in health care. $\mathrm{N}$ Engl J Med. 1989; 320: 53-6. PMid:2909878 https ://doi.org/ 10.1056/NEJM198901053200110

[10] Zhan C, Smith SR, Keyes MA, et al. How useful are voluntary medication error reports? The case of warfarin-related medication errors. Jt Comm J Qual Patient Saf. 2008; 34: 36-45. https : //doi.org/10.1016/S1553-7250(08) 34006-9

[11] Wu WH, Wang YM, Huang WT, et al. The Use of control chart to monitor and improve medication incident. Formos J Clin Pharma. 2012; 20: 301-14

[12] Leap LL. Reporting of adverse events. N Engl J Med. 2002; 347 : 1633-8. PMid:12432059 https : //doi .org/10.1056/NEJMNEJM hpr011493

[13] Shih CL, Hou SM, Hsueh YS, et al. Reporting system and the obstacle to medical incidents. Formos J Med. 2005; 9: 63.

[14] Kavanagh C. Medication governance: preventing errors and promoting patient safety. Br J Nurs. 2017; 26(3): 159-65. PMid:28185490 https://doi.org/10.12968/bjon.2017.26.3.159

[15] Barker KN, Flynn EA, Pepper GA, et al. Medication errors observed in 36 health care facilities. Arch Intern Med. 2002; 162(16): 1897903. PMid:12196090 https://doi.org/10.1001/archinte.1 62.16 .1897

[16] Tissot E, Cornette C, Limat S, et al. Observational study of potential risk factors of medication administration errors. Pharm World Sci. 2003; 25: 264-8. PMid:14689814 https://doi .org/10.1023/B: PHAR. $0000006519.44483 . \mathrm{aO}$

[17] Berdot S, Roudot M, Schramm C, et al. Interventions to reduce nurses' medication administration errors in inpatient settings: A systematic review and meta-analysis. Int J Nurs Stud. 2016; 53: 342-50. PMid:26365701 https://doi.org/10.1016/j.ijnurstu.201 5.08 .012

[18] Kavanagh C. Medication governance: preventing errors and promoting patient safety. Br J Nurs. 2017; 26(3): 159-165. PMid:28185490 https://doi.org/10.12968/bjon.2017.26.3.159
[19] Gibson T. Nurses and medication error: a discursive reading of the literature. Nurs Inq. 2001; 8: 108-17. PMid:11882209 https: //doi.org/10.1046/j.1440-1800.2001.00098.x

[20] Yazdanshenas Ghazwin M, Kavian M, Ahmadloo M et al. The association between life satisfaction and the extent of depression, anxiety and stress among Iranian nurses: a multicenter survey. Iran J Psychiatry. 2016; 11(2): 120-7. PMid:27437009

[21] Meurier CE. Understanding the nature of errors in nursing: using a model to analyse critical incident reports of errors which had resulted in an adverse or potentially adverse event. J Adv Nurs. 201; 32: 202-7. https://doi.org/10.1046/j.1365-2648.2000.01444.x

[22] Zhan C, Smith SR, Keyes MA, et al. How useful are voluntary medication error reports? The case of warfarin-related medication errors. Jt Comm J Qual Patient Saf. 2008; 34: 36-45. https : //doi.org/10.1016/S1553-7250(08) 34006-9

[23] Ko W, Kiser-Larson N. Stress Levels of Nurses in Oncology Outpatient Units. Clin J Oncol Nurs. 2016; 20(2): 158-64. PMid:26991708 https://doi.org/10.1188/16. CJON.158-164

[24] Lambert VA, Lambert CE, Ito M. Workplace stressors, ways of coping and demographic characteristics as predictors of physical and mental health of Japanese hospital nurses. Int J Nurs Stud. 2003; 41: 85-97. https://doi .org/10.1016/S0020-7489(03) 00080-4

[25] McMeekin DE, Hickman RL Jr, Douglas SL, et al. Stress and coping of critical care nurses after unsuccessful cardiopulmonary resuscitation. Am J Crit Care. 2017; 26(2): 128-135. PMid:28249865 https://doi.org/10.4037/ajcc2017916

[26] Rassin M, Kanti T, Silner D. Chronology of medication errors by nurses: accumulation of stresses and PTSD symptoms. Issues Ment Health Nurs. 2005; 26: 873-86. PMid:16203641 https: //doi.org/10.1080/01612840500184566

[27] Kiguba R, Waako P, Ndagije HB, et al. Medication error disclosure and attitudes to reporting by healthcare professionals in a sub-Saharan African setting: A Survey in Uganda. Drugs Real World Outcomes. 2015; 2(3): 273-87. PMid:26594614 https: //doi.org/10.1007/s40801-015-0032-7

[28] Kao CC, Lin YH, Lee I, et al. Development and validation of the inventory of perceptions of medication administration errors for nurses in Taiwan. J Nurs Res. 2015; 23(1): 41-6. PMid:25233070 https://doi.org/10.1097/jnr.0000000000000048

[29] Zhang Y, Long X, Chen W, et al. A concise drug alerting rule set for Chinese hospitals and its application in computerized physician order entry (CPOE). Springerplus. 2016; 5(1): 2067. PMid:27995044 https://doi .org/10.1186/s40064-016-3701-4

[30] Siebert JN, Ehrler F, Combescure C, et al. A mobile device app to reduce time to drug delivery and medication errors during simulated pediatric cardiopulmonary resuscitation: a randomized controlled trial. J Med Internet Res. 2017; 19(2): e31. https : //doi .org/10 $.2196 /$ jmir.7005

[31] Berland A, Bentsen SB. Medication errors in home care: a qualitative focus group study. J Clin Nurs. 2017. https://doi .org/10.111 $1 /$ jocn. 13745 